Nervenarzt 2021 · 92:641-642

https://doi.org/10.1007/s00115-021-01156-3

Angenommen: 27. Mai 2021

(c) Springer Medizin Verlag $\mathrm{GmbH}$, ein Teil von Springer Nature 2021

\title{
Update Borderline-Störungen - aktueller Stand und Herausforderungen
}

\author{
M. Bohus
}

Institut für Psychiatrische und Psychosomatische Psychotherapieforschung, Lehrstuhl für Psychosomatische Medizin und Psychotherapie, Zentralinstitut für Seelische Gesundheit, Mannheim, Deutschland

Mit Recht zählt die Borderline-Persönlichkeitsstörung (BPS) heute zu den schwerwiegenden psychischen Störungen (,severe mental illnesses"). Eine Lebenszeitprävalenz von etwa $3 \%[1,2]$, der frühe Beginn in der Adoleszenz, ausgeprägte psychische und soziale Belastungen, chronische Suizidalität und Selbstverletzungen sowie eine nicht unerhebliche Chronifizierungstendenz stellen Betroffene, Angehörige und auch das Gesundheitssystem vor erhebliche Herausforderungen. Studien berichten über einen Borderline-Patienten-Anteil im stationären und ambulanten Bereich für Erwachsene zwischen $12 \%$ und $22 \%$ und für Jugendliche zwischen $11 \%$ und $35 \%$ bis zu $35,6 \%$ [1].

In den zurückliegenden Jahrzehnten haben wir deutliche Fortschritte gemacht, was die Aufklärung der psychologischen und neurobiologischen Pathomechanismen betrifft. In Folge dessen wurden auch die evidenzbasierten Behandlungsmöglichkeiten erheblich verbessert. Dennoch, oder gerade deshalb stellen sich neue Herausforderungen für die Zukunft, von denen hier nur einige herausgegriffen werden sollen:

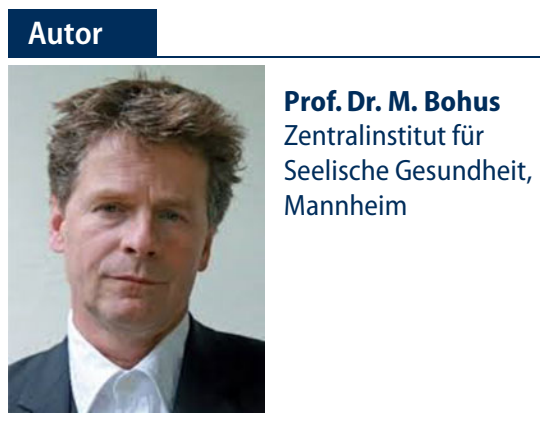

Die Versorgungslage für Jugendliche mit Borderline-Störungen in Deutschland ist prekär. Es gibt mittlerweile zwar einige Kinder- und Jugendpsychiatrien, die evidenzbasierte stationäre Behandlungsprogramme anbieten, dennoch ist das Behandlungsangebot im Vergleich zur Nachfrage so gering, dass die Jugendlichen je nach Region bis zu zwei Jahre auf ein stationäres Behandlungsangebot warten müssen, und dieses liegt dann häufig wohnortsfern.

\section{॥) Jugendliche müssen je nach Region bis zu zwei Jahre auf ambulante oder stationäre Behandlungsangebote warten}

Um die ambulante Versorgung dieser Jugendlichen ist es noch schlechter gestellt. Es gibt kaum niedergelassene Therapeuten die über eine Zusatzqualifikation in evidenzbasierten Behandlungsmethoden verfügen und bei nicht ausgebildeten Therapeuten ist die Skepsis bezüglich der Behandlung von Jugendlichen mit einer Borderline-Störung hoch. Die Vorurteile, Jugendliche mit dieser Störung seien nicht behandelbar und schlicht zu anstrengend, halten sich leider hartnäckig. Obwohl mehrere kontrollierte randomisierte Studien nachgewiesen haben, dass Jugendliche mit einer BPS erfolgreich behandelt werden können und eine Chronifizierung der Symptomatik so verhindert werden könnte, bleiben die Jugendlichen oft unversorgt und bzw. dann über längere Phasen und repetitiv auf Akutstationen, die kein spezielles Konzept für die Behandlung dieser Patienten vorweisen. Eine Hospitalisierung der Jugendlichen ist damit vorbestimmt. Viele bisweilen katastrophale Langzeitentwicklungen von BorderlineStörungen sind durchaus iatrogen induziert.

Auch im Erwachsenensektor ist die ambulante Versorgung nach wie vor unzulänglich und muss von einem mittlerweile gut strukturierten stationären Versorgungssystem unter hohem Kostenaufwand aufgefangen werden. Es fehlen Gratifizierungsangebote für Therapeuten, die über eine Zusatzausbildung in evidenzbasierten Therapieverfahren verfügen und diese dann auch bei dieser Klientel in der ambulanten Regelversorgung anwenden.

\section{》) Es fehlen Gratifizierungs- angebote für Therapeuten mit Zusatzausbildung in evidenzbasier- ten Therapien}

Unsere Kenntnisse über Borderline-Störungen fokussieren im wesentlichen auf die Alterspanne zwischen 15 und 45. Wir wissen ausgesprochen wenig über Langzeitverläufe in der späteren Lebensspanne sowie im Alter. Neuere Studien weisen auf einen Symptomwandel hin, auf eine Verschiebung der Problematik von den auffälligen Störungen der Impulskontrolle und Emotionsregulation hin zu chronischer Depressivität und ausgeprägten somatischen Erkrankungen, die dann auch zu einer deutlich reduzierte Lebenserwartung beitragen [3]. Hier scheint dringend Forschungsbedarf geboten.

Eine offene Frage bleibt die nosologische Zuordnung. Auch wenn es gelungen ist, die BPS in der ICD 11 zu verankern und diese damit nicht, wie ursprünglich geplant, der vollständigen Revision der Per- 
sönlichkeitsstörungen zum Opfer fiel, so bleibt doch offen, ob die BPS auf Dauer in diesem Diagnosespektrum sinnvoll lokalisiert ist. Angesichts der eindeutigen Datenlage hinsichtlich Traumatisierung und emotionaler Vernachlässigung drängt sich die Frage auf, ob die BPS nicht besser bei den stressassoziierten Störungen eingebunden wäre. Die Überlappungen mit der dort neu verordneten komplexen posttraumatischen Belastungsstörung (PTBS) sind doch zu augenscheinlich.

Dies sind nur einige, zugegeben etwas arbiträr herausgegriffene Fragestellungen. Auch das hier vorliegende Themenheft bietet natürlich lediglich einen Überblick über ausgewählte Aspekte zum Stand des Wissens auf dem Gebiet der BorderlineStörungen und zu den gegenwärtigen Herausforderungen, die diese Störung an das Gesundheitssystem stellt.

Die Arbeiten von J. Stoffers-Winterling und Koautoren sowie von K. Bertsch und $S$. Herpertz befassen sich mit dem gegenwärtigen Stand des Wissens über Borderline-Störungen. Sie geben einen fundierten Überblick über Nosologie, Ätiologie, Phänomenologie, neurobiologische Mechanismen und den Stand der Forschung hinsichtlich psychotherapeutischer und pharmakologischer Behandlungsoptionen.

F. Jacobi und Koautoren beleuchten auf Basis eines großen Datensatzes zum einen die erheblichen Krankheitskosten, aber auch somatische Komorbidität und Mortalitätsraten der Borderline-Persönlichkeitsstörung.

S. Hohmann und Koautoren geben einen Überblick über ein Modellprojekt zur integrativen stationären und ambulanten Langzeitbehandlung adoleszentärer Borderline-Patienten am ZI Mannheim.

Und schließlich beleuchten S. Cornelisse und Koautoren die Wirksamkeit einer störungsspezifischen Behandlung der häufigen komorbiden posttraumatischen Belastungsstörung bei Adoleszenten mit Borderline-Symptomatik.

Eine interessante Lektüre wünscht Ihnen

Ihr

M. Bohus
Korrespondenzadresse

\section{Prof. Dr. M. Bohus}

Institut für Psychiatrische und Psychosomatische Psychotherapieforschung, Lehrstuhl für Psychosomatische Medizin und Psychotherapie, Zentralinstitut für Seelische Gesundheit

J 5, 68159 Mannheim, Deutschland martin.bohus@zi-mannheim.de

Interessenkonflikt. M. Bohus ist wissenschaftlicher Berater der Oberberg Kliniken sowie der Schlemmer Klinik und des CIP München. Er hält Anteile an der AWP Freiburg, schreibt Bücher und Behandlungsmanuale zum Thema DBT und DBT-PTSD. Er hältzudem Vorträge und Workshops zu dieser Thematik.

\section{Literatur}

1. Ellison WD, Rosenstein LK, Morgan TA, Zimmerman M (2018) Community and clinical epidemiology of borderline personality disorder. Psychiatr Clin North Am 41:561-573

2. Jørgensen MS, Storebø OJ, Stoffers-Winterling JM, Faltinsen E, Todorovac A, Simonsen E (2021) Psychological therapies for adolescents with borderline personality disorder (BPD) or BPD features - a systematic review of randomized clinical trials with meta-analysis and Trial Sequential Analysis. PLoSONE 16(1):e245331. https://doi.org/ 10.1371/journal.pone.0245331

3. Schneider F, Erhart M, Hewer W et al (2019) Mortality and medical comorbidity in the severely mentally ill: a German registry study. Dtsch Arztebl Int 116:405

\section{Springer Medizin Podcast \\ Medizin für Gesundheitsprofis}

Der Podcast von SpringerMedizin.de geht seit Juli 2020 spannenden Fragen aus der Welt der Medizin nach immer freitags erweitert eine neue Folge das bereits bestehende OnlineAngebot.

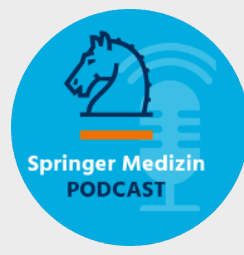

„Mit unserem

Podcast möchten wir all jene ansprechen, die sich auf medizinische Themen in einer, gewissen

Flughöhe' einlassen möchten", erklärt Dr. Erik Heintz, Chefredakteur von SpringerMedizin.de das neue Format. "Gemeint sind damit Menschen, die sich nicht mit medizinischem Halbwissen zufrieden geben und gerne mehr wissen möchten. Daher legen unsere Redakteurinnen und Redakteure in München und Heidelberg vor allem auf den inhaltlichen Anspruch großen Wert. Unter Bezugnahme auf neue Studien, neue Erkenntnisse und praxisrelevantes Wissen bereiten wir jeden Podcast gründlich vor. Die Themen drehen sich um Gesundheit, Krankheit, Diagnostik, Therapie und Prävention, zu denen wir unsere Fachexpertinnen und Fachexperten aus verschiedenen medizinischen Fachgebieten hinzuziehen."

So entstehen abwechslungsreiche Folgen, die sowohl detailliert Leitlinien abarbeiten, der Evidenz von neuen Studienergebnissen auf den Grund gehen, aber auch die Hintergründe zu Erkrankungen und Therapien aufschlüsseln - sodass einerseits die ärztliche Praxis erleichtert und konkrete Tipps für die alltägliche Arbeit vermittelt werden, andererseits aber auch der Horizont über das eigene Fachgebiet hinaus erweitert werden kann.

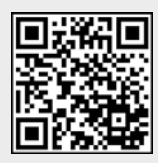

www.springermedizin.de/podcast 